\title{
General decay and blow-up of solutions for a nonlinear viscoelastic wave equation with strong damping
}

\section{Qian Li ${ }^{1 *}$ (D) and Luofei He'}

\section{"Correspondence:}

2016102010017@whu.edu.cn

1 School of Mathematics and

Statistics, Wuhan University, Wuhan, China

\section{照 Springer}

\begin{abstract}
This article is concerned with the decay and blow-up properties of a nonlinear viscoelastic wave equation with strong damping. We first show a local existence theorem. Then, we prove the global existence of solutions and establish a general decay rate estimate. Finally, we show the finite time blow-up result for some solutions with negative initial energy and positive initial energy.
\end{abstract}

Keywords: Viscoelastic wave equation; Strong damping; General decay; Blow up

\section{Introduction}

In this work we investigate the decay and blow-up properties of the nonlinear viscoelastic wave equation of the form:

$$
\begin{cases}u_{t t}-\Delta u+\int_{0}^{t} g(t-\tau) \Delta u(\tau) d \tau-\Delta u_{t}+u_{t}=u|u|^{p-2}, & \text { in } \Omega \times(0, \infty), \\ u(x, t)=0, & \text { on } \partial \Omega \times(0, \infty), \\ u(x, 0)=u_{0}(x), \quad u_{t}(x, 0)=u_{1}(x), & \text { in } \Omega,\end{cases}
$$

where $\Omega \subset \mathbb{R}^{n}$ is bounded domains with smooth boundary $\partial \Omega$. Problems of this type have been investigated by many authors, and some results in connection with existence and nonexistence have been established. For example, Berrimi and Messaoudi [1] studied the following viscoelastic equation:

$$
\begin{cases}u_{t t}-\Delta u+\int_{0}^{t} g(t-\tau) \Delta u(\tau) d \tau=u|u|^{\gamma}, & \text { in } \Omega \times(0, \infty), \\ u(x, t)=0, & \text { on } \partial \Omega \times(0, \infty), \\ u(x, 0)=u_{0}(x), \quad u_{t}(x, 0)=u_{1}(x), & \text { in } \Omega,\end{cases}
$$

where $\gamma>0$. The authors established the local existence and global existence theorems and showed that the solution energy exponentially or polynomially decays. Later, Messaoudi [17] improved the results of [1], he established a general decay result. Inspired by the ideas of Messaoudi [17] and [18], Han and Wang [10] investigated a nonlinear viscoelastic equation with the dispersive term $\Delta u_{t t}$ by modifying the perturbed energy functional; they also obtained that the solution energy is general decay. Recently, Guesmia et al. [8] combined

(c) The Author(s) 2018. This article is distributed under the terms of the Creative Commons Attribution 4.0 International License (http://creativecommons.org/licenses/by/4.0/), which permits unrestricted use, distribution, and reproduction in any medium, provided you give appropriate credit to the original author(s) and the source, provide a link to the Creative Commons license, and indicate if changes were made. 
the techniques given in [17] with the character of Kirchhoff equation and obtained the optimal decay rate estimate of solution energy. For the case of wave equation with nonlinear boundary damping and source terms, Vitillaro [21] established the local and global existence of solutions under reasonable conditions on the initial data. In [3], Cavalcanti et al. obtained both well-posedness and the optimal decay rate estimate for solutions.

In article [6], Gazzola and Squassina discussed the following viscoelastic equation with strong damping term $\Delta u_{t}$ :

$$
\begin{cases}u_{t t}-\Delta u-\omega \Delta u_{t}+\mu u_{t}=u|u|^{p-2}, & \text { in } \Omega \times(0, \infty), \\ u(x, t)=0, & \text { on } \partial \Omega \times(0, \infty), \\ u(x, 0)=u_{0}(x), \quad u_{t}(x, 0)=u_{1}(x), & \text { in } \Omega,\end{cases}
$$

where $p>2, \omega, \mu>0$. The authors established the global existence theorem and proved that the global solution is uniformly bounded. They also constructed the finite time blowup of solutions for low initial energy or arbitrarily high initial energy. When the linear damping term is replaced by nonlinear damping term in equation (1.2), Chen and Liu [5] obtained a global existence theorem, uniform decay rate estimate, and exponential growth for the solutions.

In paper [2], Cavalcanti et al. dealt with the following problem:

$$
\begin{cases}\left|u_{t}\right|^{\rho} u_{t t}-\Delta u-\Delta u_{t t}+\int_{0}^{t} g(t-\tau) \Delta u(\tau) d \tau-\gamma \Delta u_{t}=0, & \text { in } \Omega \times(0, \infty), \\ u(x, t)=0, & \text { on } \partial \Omega \times(0, \infty), \\ u(x, 0)=u_{0}(x), \quad u_{t}(x, 0)=u_{1}(x), & \text { in } \Omega,\end{cases}
$$

under reasonable conditions on $g$ and $\gamma$, the authors established the global existence result for $\gamma \geq 0$ and the exponential decay result for $\gamma>0$. Cavalcanti et al. [4] discussed equation (1.3) for $\rho \geq 0, \gamma \geq 0$ and obtained that the energy decays to zero with the decay rate which is dominated by the solutions of the ODE quantifying the conduct of $g(t)$.

For the finite time blow-up, Messaoudi [15] studied the following problem:

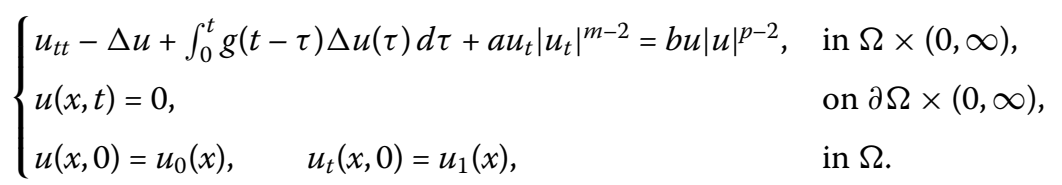

He showed that the solution blows up in finite time when the initial energy is negative and $p>m$ and the solution exists globally for $m \geq p$. In [16], Messaoudi extended the blow-up result to certain situations in which the initial energy is positive. Later, Song [19] proved the finite time blow-up of solutions whose initial data have arbitrarily high initial energy. It is worth mentioning some other literatures concerning existence and nonexistence of wave equation, namely $[7,9,11,13,14,20]$ and the references therein.

At the present time, less results are investigated for the wave equation with strong damping term and many problems are unsolved (see [6]). So, in this paper, we study a nonlinear viscoelastic wave equation with strong damping. We first show a local existence theorem. Then, we prove the global existence of solutions and establish a general decay rate estimate. Finally, we show the finite time blow-up result for some solutions with negative initial energy and positive initial energy. 
This article is organized as follows. In Sect. 2, we give some preliminaries. In Sect. 3, we prove the local existence and uniqueness of solutions for problem (1.1). Then, the general decay of the solutions is considered in Sect. 4. In the last section, we discuss the blow-up phenomenon for the equation.

\section{Preliminaries}

We begin with some materials needed in the proof of the main results. We first recall the following assumptions as in [17]:

(H1) $g: R_{+} \rightarrow R_{+}$is a nonincreasing and bounded $C^{1}$ function satisfying

$$
g(0)>0, \quad 1-\int_{0}^{\infty} g(\tau) d \tau=l>0 .
$$

(H2) There exists a positive differentiable function $\xi(t)$ such that

$$
g^{\prime}(t) \leq-\xi(t) g(t), \quad t \geq 0
$$

and

$$
\left|\frac{\xi^{\prime}(t)}{\xi(t)}\right| \leq k, \quad \xi(t)>0, \xi^{\prime}(t) \leq 0, \quad \forall t>0, \quad \int_{0}^{+\infty} \xi(t) d t=+\infty
$$

(H3) For the nonlinear term, we let

$$
2<p \leq \frac{2 n}{n-2}, \quad \text { if } n \geq 3 ; \quad 2<p<\infty, \quad \text { if } n=1,2 .
$$

Remark 2.1 Since $\xi$ is a nonincreasing function, then $\xi(t) \leq \xi(0)=M$.

We will use the embedding $H_{0}^{1}(\Omega) \hookrightarrow L^{s}(\Omega)$ for $2 \leq s \leq 2 n /(n-2)$ if $n \geq 3$ or $s \geq 2$ if $n=1,2$; and $L^{r}(\Omega) \hookrightarrow L^{s}(\Omega)$ for $s<r$, and we will use the same embedding constant denoted by $C_{*}$ such that

$$
\|u\|_{s} \leq C_{*}\|\nabla u\|_{2}, \quad\|u\|_{s} \leq C_{*}\|u\|_{r} .
$$

For our aim, we use the following functionals:

$$
\begin{aligned}
& I(t)=I(u(t))=\left(1-\int_{0}^{t} g(\tau) d \tau\right)\|\nabla u(t)\|_{2}^{2}+(g \circ \nabla u)(t)-\|u(t)\|_{p}^{p}, \\
& J(t)=J(u(t))=\frac{1}{2}\left(1-\int_{0}^{t} g(\tau) d \tau\right)\|\nabla u(t)\|_{2}^{2}+\frac{1}{2}(g \circ \nabla u)(t)-\frac{1}{p}\|u(t)\|_{p}^{p}, \\
& E(t)=E\left(u(t), u_{t}(t)\right)=J(t)+\frac{1}{2}\left\|u_{t}\right\|_{2}^{2},
\end{aligned}
$$

where

$$
(g \circ v)(t)=\int_{0}^{t} g(t-\tau)\|v(t)-v(\tau)\|_{2}^{2} d \tau .
$$


Lemma 2.2 If $(\mathrm{H} 1),(\mathrm{H} 2)$, (H3) hold and $\left(u_{0}, u_{1}\right) \in H_{0}^{1}(\Omega) \times L^{2}(\Omega)$, $u$ is the solution of $(1.1)$, then the energy functional $E(t)$ satisfies

$$
E^{\prime}(t)=\frac{1}{2}\left(g^{\prime} \circ \nabla u\right)(t)-\frac{1}{2} g(t)\|\nabla u(t)\|_{2}^{2}-\left\|\nabla u_{t}\right\|_{2}^{2}-\left\|u_{t}\right\|_{2}^{2} \leq 0
$$

for $\forall t \in[0, T]$.

Proof Multiplying (1.1) by $u_{t}$ and integrating over $\Omega$, we obtain

$$
\begin{aligned}
\frac{d}{d t} & \left\{\frac{1}{2} \int_{\Omega}\left|u_{t}\right|^{2} d x+\frac{1}{2} \int_{\Omega}|\nabla u|^{2} d x-\frac{1}{p} \int_{\Omega}|u|^{p} d x\right\} \\
& -\int_{0}^{t} g(t-\tau) \int_{\Omega} \nabla u_{t}(t) \cdot \nabla u(\tau) d x d \tau=-\int_{\Omega}\left|\nabla u_{t}\right|^{2} d x-\int_{\Omega}\left|u_{t}\right|^{2} d x .
\end{aligned}
$$

For the last term on the left-hand side of (2.4), we get

$$
\begin{aligned}
\int_{0}^{t} g(t-\tau) \int_{\Omega} \nabla u_{t}(t) \cdot \nabla u(\tau) d x d \tau \\
=\int_{0}^{t} g(t-\tau) \int_{\Omega} \nabla u_{t}(t) \cdot[\nabla u(\tau)-\nabla u(t)] d x d \tau \\
\quad+\int_{0}^{t} g(t-\tau) \int_{\Omega} \nabla u_{t}(t) \cdot \nabla u(t) d x d \tau \\
=-\frac{1}{2} \int_{0}^{t} g(t-\tau)\left(\frac{d}{d t} \int_{\Omega}|\nabla u(\tau)-\nabla u(t)|^{2} d x\right) d \tau \\
\quad+\int_{0}^{t} g(\tau)\left(\frac{d}{d t} \frac{1}{2} \int_{\Omega}|\nabla u(t)|^{2} d x\right) d \tau \\
=-\frac{1}{2} \frac{d}{d t}\left[\int_{0}^{t} g(t-\tau) \int_{\Omega}|\nabla u(\tau)-\nabla u(t)|^{2} d x d \tau\right] \\
\quad+\frac{1}{2} \frac{d}{d t}\left[\int_{0}^{t} g(\tau) \int_{\Omega}|\nabla u(t)|^{2} d x d \tau\right] \\
\quad+\frac{1}{2} \int_{0}^{t} g^{\prime}(t-\tau) \int_{\Omega}|\nabla u(\tau)-\nabla u(t)|^{2} d x d \tau \\
\quad-\frac{1}{2} g(t) \int_{\Omega}|\nabla u(t)|^{2} d x .
\end{aligned}
$$

Inserting (2.5) into (2.4), we obtain

$$
\begin{aligned}
\frac{d}{d t}\{ & \left.\frac{1}{2} \int_{\Omega}\left|u_{t}\right|^{2} d x+\frac{1}{2} \int_{\Omega}|\nabla u|^{2} d x-\frac{1}{p} \int_{\Omega}|u|^{p} d x\right\} \\
& -\frac{1}{2} \frac{d}{d t}\left[\int_{0}^{t} g(\tau) d \tau\|\nabla u(t)\|_{2}^{2}\right]+\frac{1}{2} \frac{d}{d t}\left[\int_{0}^{t} g(t-\tau) \int_{\Omega}|\nabla u(\tau)-\nabla u(t)|^{2} d x d \tau\right] \\
= & -\int_{\Omega}\left|\nabla u_{t}\right|^{2} d x-\int_{\Omega}\left|u_{t}\right|^{2} d x+\frac{1}{2}\left(g^{\prime} \circ \nabla u\right)(t)-\frac{1}{2} g(t)\|\nabla u(t)\|_{2}^{2} \leq 0
\end{aligned}
$$


Lemma 2.3 If (H3) holds, then there exists a positive constant $C>1$ such that

$$
\|u\|_{p}^{s} \leq C\left(\|\nabla u\|_{2}^{2}+\|u\|_{p}^{p}\right), \quad 2 \leq s \leq p
$$

for any $u$ being a solution of (1.1) on $[0, T]$.

Proof If $\|u\|_{p} \leq 1$, then $\|u\|_{p}^{s} \leq\|u\|_{p}^{2} \leq C\|\nabla u\|_{2}^{2}$ by using Sobolev embedding theorems. If $\|u\|_{p}>1$, then $\|u\|_{p}^{s} \leq\|u\|_{p}^{p}$. Therefore (2.7) follows.

We set

$$
H(t):=-E(t)
$$

and use $C$ to denote a general positive constant depending on $\Omega$ only. As a result of (2.2) and (2.7), we have the following.

Corollary 2.4 Let the assumption of the above lemma hold. Then we have the following:

$$
\|u\|_{p}^{s} \leq C\left(H(t)+\left\|u_{t}\right\|_{2}^{2}+\|\nabla u\|_{2}^{2}+(g \circ \nabla u)(t)\right), \quad \forall t \in[0, T]
$$

for any $u \in H_{0}^{1}(\Omega)$ and $2 \leq s \leq p$.

Proof Using (H1) and (2.2) leads to

$$
\begin{aligned}
\frac{1}{p}\|u(t)\|_{p}^{p} & \leq-E(t)+\frac{1}{2}\left\|u_{t}\right\|_{2}^{2}+\frac{1}{2}\left(1-\int_{0}^{\infty} g(\tau) d \tau\right)\|\nabla u\|_{2}^{2}+\frac{1}{2}(g \circ \nabla u)(t) \\
& \leq H(t)+\frac{1}{2}\left\|u_{t}\right\|_{2}^{2}+\frac{1}{2}\left(1-\int_{0}^{\infty} g(\tau) d \tau\right)\|\nabla u\|_{2}^{2}+\frac{1}{2}(g \circ \nabla u)(t) .
\end{aligned}
$$

Finally, a combination of (2.7) and (2.9) gives the needed result.

\section{Local existence}

In this section, the aim is to establish the local existence result for (1.1). For this goal, we first discuss a related linear problem. Then, by using the contraction mapping theorem, we obtain the existence of solutions to the nonlinear problem. For $v$ given, the related linear problem is of the form:

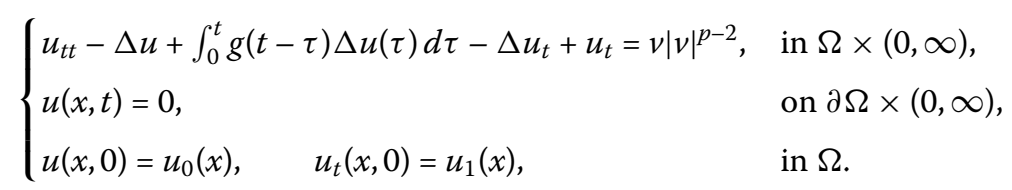

Similar to the proof in [12], we can get the following lemma. 
Lemma 3.1 Suppose $v \in C\left([0, T] ; C_{0}^{\infty}(\Omega)\right)$ and $u_{0}, u_{1} \in C_{0}^{\infty}(\Omega)$, then problem (3.1) has a unique solution $u$ satisfying

$$
\begin{aligned}
& u \in L^{\infty}\left((0, T) ; H_{0}^{1}(\Omega) \cap H^{2}(\Omega)\right), \\
& u_{t} \in L^{\infty}\left((0, T) ; H_{0}^{1}(\Omega)\right), \\
& u_{t t} \in L^{\infty}\left((0, T) ; L^{2}(\Omega)\right) .
\end{aligned}
$$

Next, we prove the existence of solutions to equation (3.1) when the initial data is less regular.

Lemma 3.2 If (H3) holds, then given any $\left(u_{0}, u_{1}\right) \in H_{0}^{1}(\Omega) \times L^{2}(\Omega)$ and $v \in C([0, T]$; $H_{0}^{1}(\Omega)$ ), problem (3.1) has a unique weak solution

$$
u \in C\left([0, T] ; H_{0}^{1}(\Omega)\right), \quad u_{t} \in C\left([0, T] ; L^{2}(\Omega)\right)
$$

Proof We approximate $u_{0}, u_{1}$ by sequences $\left(u_{0 n}\right),\left(u_{1 n}\right)$ in $C_{0}^{\infty}(\Omega)$, and $v$ by a sequence $\left(v_{n}\right)$ in $C\left([0, T] ; C_{0}^{\infty}(\Omega)\right)$. Then from Lemma 3.1 we can obtain a solution $\left(u_{n}\right)$ satisfying:

$$
\begin{cases}u_{n t t}-\Delta u_{n}+\int_{0}^{t} g(t-\tau) \Delta u_{n}(\tau) d \tau-\Delta u_{n t}+u_{n t}=v_{n}\left|v_{n}\right|^{p-2}, & \text { in } \Omega \times(0, \infty), \\ u_{n}(x, t)=0, & \text { on } \partial \Omega \times(0, \infty), \\ u_{n}(x, 0)=u_{0 n}(x), & u_{n t}(x, 0)=u_{1 n}(x),\end{cases}
$$

and satisfying (3.2). Now we prove that the sequence $\left(u_{n}\right)$ is Cauchy in

$$
W:=\left\{u: u \in C\left([0, T] ; H_{0}^{1}(\Omega)\right) \cap C^{1}\left([0, T] ; L^{2}(\Omega)\right), u_{t} \in C\left([0, T] ; L^{2}(\Omega)\right)\right\},
$$

with the defined norm

$$
\|u\|_{W}^{2}:=\max _{0 \leq t \leq T} \frac{1}{2}\left(\left\|u_{t}\right\|_{2}^{2}+l\|\nabla u\|_{2}^{2}\right)+\int_{0}^{T}\left\|\nabla u_{t}\right\|_{2}^{2} d \tau+\int_{0}^{T}\left\|u_{t}\right\|_{2}^{2} \leq M^{2} .
$$

For this purpose, we let $U=u_{n}-u_{n^{\prime}}, V=v_{n}-v_{n^{\prime}}$, then $U$ satisfies

$$
\left\{\begin{array}{l}
U_{t t}-\Delta U+\int_{0}^{t} g(t-\tau) \Delta U(\tau) d \tau-\Delta U_{t}+U_{t} \\
\quad=v_{n}\left|v_{n}\right|^{p-2}-v_{n^{\prime}}\left|v_{n^{\prime}}\right|^{p-2}, \quad x \in \Omega, t>0 \\
U(x, t)=0, \quad x \in \partial \Omega, t \geq 0, \\
U(x, 0)=U_{0}(x)=u_{0 n}-u_{0 n^{\prime}}, \quad U_{t}(x, 0)=U_{1}(x)=u_{1 n}-u_{1 n^{\prime}}, \quad x \in \Omega .
\end{array}\right.
$$

Multiplying (3.6) by $U_{t}$ and integrating over $(0, t) \times \Omega$, we obtain

$$
\begin{gathered}
\frac{1}{2}\left[\left\|U_{t}\right\|_{2}^{2}+\left(1-\int_{0}^{t} g(\tau) d \tau\right)\|\nabla U\|_{2}^{2}\right]-\frac{1}{2} \int_{0}^{t}\left(g^{\prime} \circ \nabla U\right)(\tau) d \tau+\int_{0}^{t}\left\|\nabla U_{t}\right\|_{2}^{2} d \tau \\
+\frac{1}{2}(g \circ \nabla U)(t)+\frac{1}{2} \int_{0}^{t} \int_{\Omega} g(\tau)|\nabla U(\tau)|^{2} d x d \tau+\int_{0}^{t}\left\|U_{t}\right\|_{2}^{2} d \tau \\
=\frac{1}{2}\left(\left\|U_{1}\right\|_{2}^{2}+\left\|\nabla U_{0}\right\|_{2}^{2}\right)+\int_{0}^{t} \int_{\Omega}\left(v_{n}\left|v_{n}\right|^{p-2}-v_{n^{\prime}}\left|v_{n^{\prime}}\right|^{p-2}\right) U_{t} d x d \tau .
\end{gathered}
$$


We estimate the second term on the right-hand side of (3.7) as follows:

$$
\begin{aligned}
& \int_{\Omega}\left(v_{n}\left|v_{n}\right|^{p-2}-v_{n^{\prime}}\left|v_{n^{\prime}}\right|^{p-2}\right) U_{t} d x \\
& \quad \leq C\left\|U_{t}\right\|_{2}\|V\|_{\frac{2 n}{n-2}}\left(\left\|v_{n}\right\|_{n(p-2)}^{p-2}+\left\|v_{n^{\prime}}\right\|_{n(p-2)}^{p-2}\right) \\
& \quad \leq C\left\|U_{t}\right\|_{2}\|\nabla V\|_{2}\left(\left\|\nabla v_{n}\right\|_{2}^{p-2}+\left\|\nabla v_{n^{\prime}}\right\|_{2}^{p-2}\right),
\end{aligned}
$$

where $C$ is a constant. Using $(\mathrm{H} 1),(\mathrm{H} 2)$ and the following fact:

$$
-\frac{1}{2} \int_{0}^{t}\left(g^{\prime} \circ \nabla U\right)(\tau) d \tau+\frac{1}{2}(g \circ \nabla U)(t)+\frac{1}{2} \int_{0}^{t} \int_{\Omega} g(\tau)|\nabla U(\tau)|^{2} d x d \tau \geq 0,
$$

by estimating (3.7), we obtain

$$
\begin{aligned}
& \frac{1}{2}\left[\left\|U_{t}\right\|_{2}^{2}+l\|\nabla U\|_{2}^{2}\right]+\int_{0}^{t}\left\|\nabla U_{t}\right\|_{2}^{2} d \tau+\int_{0}^{t}\left\|U_{t}\right\|_{2}^{2} d \tau \\
& \quad \leq \frac{1}{2}\left(\left\|U_{1}\right\|_{2}^{2}+\left\|\nabla U_{0}\right\|_{2}^{2}\right)+C \int_{0}^{t}\left\|U_{t}\right\|_{2}\|\nabla V\|_{2}\left(\left\|\nabla v_{n}\right\|_{2}^{p-2}+\left\|\nabla v_{n^{\prime}}\right\|_{2}^{p-2}\right) d \tau \\
& \quad \leq \frac{1}{2}\left(\left\|U_{1}\right\|_{2}^{2}+\left\|\nabla U_{0}\right\|_{2}^{2}\right)+\Gamma \int_{0}^{t}\left\|U_{t}\right\|_{2}\|\nabla V\|_{2} d \tau,
\end{aligned}
$$

where $\Gamma>0$ is a constant depending on $\Omega, l, \gamma$ and the radius of the ball in $C\left([0, T] ; H_{0}^{1}(\Omega)\right)$ containing $\left(v_{n}\right)$ and $\left(v_{n^{\prime}}\right)$. By employing Gronwall's and Young's inequalities to the second term of (3.9), we can get

$$
\|U\|_{W}^{2} \leq \Gamma\left(\left\|U_{1}\right\|_{2}^{2}+\left\|\nabla U_{0}\right\|_{2}^{2}\right)+\Gamma T\|V\|_{W}^{2}
$$

Since $\left(u_{0 n}\right)$ is Cauchy in $H_{0}^{1}(\Omega),\left(u_{1 n}\right)$ is Cauchy in $L^{2}(\Omega)$, and $\left(v_{n}\right)$ is Cauchy in $C([0, T]$; $H_{0}^{1}(\Omega)$ ), we obtain that $\left(u_{n}\right)$ is Cauchy in $W$, then $u_{n}$ converges to a limit $u$ in $W$. Now, we prove that the limit $u$ is a weak solution of (3.1). Multiplying equation (3.4) by $\theta \in$ $H_{0}^{1}(\Omega) \cap L^{2}(\Omega)$, we obtain

$$
\begin{aligned}
& \frac{d}{d t}\left(u_{n t}, \theta\right)+\int_{\Omega} \nabla u_{n} \nabla \theta d x-\int_{0}^{t} \int_{\Omega} g(t-\tau) \nabla u_{n} \cdot \nabla \theta d \tau d x+\int_{\Omega} \nabla u_{n t} \nabla \theta d x \\
& \quad+\int_{\Omega} u_{n t} \theta d x=\int_{\Omega}\left|v_{n}\right|^{p-2} v_{n} \theta d x .
\end{aligned}
$$

When $n \rightarrow \infty$, we know that $\left(\nabla u_{n}, \nabla \theta\right) \rightarrow(\nabla u, \nabla \theta), \int_{\Omega}\left|v_{n}\right|^{p-2} v_{n} \theta d x \rightarrow \int_{\Omega}|v|^{p-2} v \theta d x$ in $C[0, T]$, and $\int_{0}^{t} \int_{\Omega} g(t-\tau) \nabla u_{n} \cdot \nabla \theta d \tau d x \rightarrow \int_{0}^{t} \int_{\Omega} g(t-\tau) \nabla u \cdot \nabla \theta d \tau d x, \int_{\Omega} \nabla u_{n t} \nabla \theta d x \rightarrow$ $\int_{\Omega} \nabla u_{t} \nabla \theta d x, \int_{\Omega} u_{n t} \theta d x \rightarrow \int_{\Omega} u_{t} \theta d x$ in $L^{1}(0, T)$, then (3.11) proves that $\lim _{n \rightarrow \infty}\left(u_{n t}, \theta\right)=$ $\left(u_{t}, \theta\right)$ is an absolutely continuous function, so $u$ is a weak solution. To show the uniqueness property, we let $v^{1}, v^{2}$ and $u^{1}, u^{2}$ be the corresponding solution of (3.1). Take $U=u^{1}-u^{2}$, 
we have

$$
\begin{aligned}
& \frac{1}{2}\left(\left\|U_{t}\right\|_{2}^{2}+l\|\nabla U\|_{2}^{2}\right)-\frac{1}{2} \int_{0}^{t}\left(g^{\prime} \circ \nabla U\right)(\tau) d \tau+\int_{0}^{t}\left\|\nabla U_{t}\right\|_{2}^{2} d \tau \\
& \quad+\frac{1}{2}(g \circ \nabla U)(t)+\frac{1}{2} \int_{0}^{t} \int_{\Omega} g(\tau)|\nabla U(\tau)|^{2} d x d \tau+\int_{0}^{t}\left\|U_{t}\right\|_{2}^{2} d \tau \\
& =\int_{0}^{t} \int_{\Omega}\left(v^{1}\left|v^{1}\right|^{p-2}-v^{2}\left|v^{2}\right|^{p-2}\right) U_{t} d x d \tau .
\end{aligned}
$$

Assume $v^{1}=v^{2}$, then (3.12) proves that $U=0$ and the solution is unique.

Next, we state and prove the local existence result theorem.

Theorem 3.3 If $u_{0} \in H_{0}^{1}(\Omega), u_{1} \in L^{2}(\Omega)$ and $H(3)$ holds, then equation (1.1) has a unique weak solution $u \in W$ for $T$ small enough.

Proof For $M>0$ large and $T>0$, we define a class of functions $Z(M, T)$ consisting of all functions $w$ in $W$ satisfying the initial data of (1.1) and $\|w\|_{W} \leq M^{2}$. We also define the map $f$ from $Z(M, T)$ into $W$ by $u:=f(w)$.

We will show that $f$ is a contraction from $Z(M, T)$ into itself. Multiplying (3.1) by $u_{t}$ and integrating over $(0, t) \times \Omega$, we obtain

$$
\|u\|_{W}^{2} \leq C\left(\left\|u_{1}\right\|_{2}^{2}+\left\|\nabla u_{0}\right\|_{2}^{2}\right)+C M^{p-1} T\|u\|_{W},
$$

where $C$ is independent of $M$. Choosing $M$ large enough and $T$ small enough, we get $u$ satisfying $\|u\|_{W} \leq M^{2}$, i.e., $u \in Z(M, T)$. This proves that $f$ maps $Z(M, T)$ into itself.

Next, we prove that $f$ is a contraction. For this aim, we let $U=u-\bar{u}$ and $V=v-\bar{v}$, where $u=f(v)$ and $\bar{u}=f(\bar{v})$, then $U$ satisfies

$$
\left\{\begin{array}{l}
U_{t t}-\Delta U+\int_{0}^{t} g(t-\tau) \Delta U(\tau) d \tau-\Delta U_{t}+U_{t} \\
=v|v|^{p-2}-\bar{v}|\bar{v}|^{p-2}, \quad x \in \Omega, t>0 \\
U(x, t)=0, \quad x \in \partial \Omega, t \geq 0 \\
U(x, 0)=U_{t}(x, 0)=0, \quad x \in \Omega .
\end{array}\right.
$$

Similar to the proof of (3.9), we get

$$
\begin{gathered}
\frac{1}{2}\left(\left\|U_{t}\right\|_{2}^{2}+l\|\nabla u\|_{2}^{2}\right)+\int_{0}^{t}\left\|\nabla U_{t}\right\|_{2}^{2} d \tau+\int_{0}^{t}\left\|U_{t}\right\|_{2}^{2} d \tau \\
\leq C \int_{0}^{t}\left\|U_{t}\right\|_{2}\|\nabla V\|_{2}\left(\|\nabla v\|_{2}^{p-2}+\|\nabla \bar{\nu}\|_{2}^{p-2}\right) d \tau .
\end{gathered}
$$

Thus we get

$$
\|U\|_{W} \leq C T M^{p-2}\|V\|_{W}
$$

We let $T$ small enough such that $C T M^{p-2} \leq \frac{1}{2}$. Then from (3.16) we can get that $f$ is a contraction in $Z(M, T)$. By using the contraction mapping principle, we can obtain that there exists a unique $u$ satisfying $u=f(u)$. Then it is the solution of (1.1). 


\section{Decay of global solution}

In this section we state and prove the general decay result for global solutions. Firstly, we establish the global existence theorem.

Lemma 4.1 If (H1), (H2), (H3) hold and $\left(u_{0}, u_{1}\right) \in H_{0}^{1}(\Omega) \times L^{2}(\Omega)$ such that

$$
\beta=\frac{C_{*}^{p}}{l}\left(\frac{2 p}{l(p-2)} E(0)\right)^{(p-2) / 2}<1, \quad I\left(u_{0}\right)>0,
$$

then $I(u(t))>0$ for $\forall t>0$. Here $C_{*}$ is given in (2.1).

Proof For $I\left(u_{0}\right)>0$, then there is $T_{m}<T$ such that $I(u(t)) \geq 0$ for $\forall t \in\left[0, T_{m}\right)$. So, we get

$$
\begin{aligned}
J(t) & =\frac{1}{2}\left(1-\int_{0}^{t} g(\tau) d \tau\right)\|\nabla u(t)\|_{2}^{2}+\frac{1}{2}(g \circ \nabla u)(t)-\frac{1}{p}\|u(t)\|_{p}^{p} \\
& =\frac{p-2}{2 p}\left(1-\int_{0}^{t} g(\tau) d \tau\right)\|\nabla u(t)\|_{2}^{2}+\frac{p-2}{2 p}(g \circ \nabla u)(t)+\frac{1}{p} I(u(t)) \\
& \geq \frac{p-2}{2 p}\left\{\left(1-\int_{0}^{t} g(\tau) d \tau\right)\|\nabla u(t)\|_{2}^{2}+(g \circ \nabla u)(t)\right\}, \quad \forall t \in\left[0, T_{m}\right) .
\end{aligned}
$$

By using (H1), (2.2), (2.3), and (4.2), we arrive at

$$
\begin{aligned}
l\|\nabla u(t)\|_{2}^{2} & \leq\left(1-\int_{0}^{t} g(\tau) d \tau\right)\|\nabla u(t)\|_{2}^{2} \leq \frac{2 p}{p-2} J(t) \\
& \leq \frac{2 p}{p-2} E(t) \\
& \leq \frac{2 p}{p-2} E(0), \quad \forall t \in\left[0, T_{m}\right) .
\end{aligned}
$$

By combining (H1), (2.1), (4.1) with (4.3), we get

$$
\begin{aligned}
\|u(t)\|_{p}^{p} & \leq C_{*}^{p}\|\nabla u(t)\|_{2}^{p} \\
& \leq \frac{C_{*}^{p}}{l}\|\nabla u(t)\|_{2}^{p-2} l\|\nabla u(t)\|_{2}^{2} \\
& \leq \frac{C_{*}^{p}}{l}\left[\frac{2 p}{l(p-2)} E(0)\right]^{(p-2) / 2} l\|\nabla u(t)\|_{2}^{2} \\
& \leq \beta l\|\nabla u(t)\|_{2}^{2} \\
& <\left(1-\int_{0}^{t} g(\tau) d \tau\right)\|\nabla u(t)\|_{2}^{2} .
\end{aligned}
$$

Therefore,

$$
I(t)=\left(1-\int_{0}^{t} g(s) d s\right)\|\nabla u(t)\|_{2}^{2}+(g \circ \nabla u)(t)-\|u(t)\|_{p}^{p}>0
$$

for $\forall t \in\left[0, T_{m}\right)$. Repeating the process and using the fact that

$$
\lim _{t \rightarrow T_{m}} \frac{C_{*}^{p}}{l}\left(\frac{2 p}{l(p-2)} E(0)\right)^{(p-2) / 2} \leq \beta<1,
$$


$T_{m}$ is extended to $T$.

Theorem 4.2 If $\left(u_{0}, u_{1}\right) \in H_{0}^{1}(\Omega) \times L^{2}(\Omega)$ and satisfies (4.1), and suppose (H1), (H2), (H3) hold, then the solution is global and bounded.

Proof The aim is to show that

$$
\|\nabla u(t)\|_{2}^{2}+\left\|u_{t}(t)\right\|_{2}^{2}
$$

is bounded independently of $t$. For this goal, we use Lemma 4.1 and (2.2) to obtain

$$
\begin{aligned}
E(0) & \geq E(t)=J(t)+\frac{1}{2}\left\|u_{t}(t)\right\|_{2}^{2} \\
& \geq \frac{p-2}{2 p}\left\{\left(1-\int_{0}^{t} g(s) d s\right)\|\nabla u(t)\|_{2}^{2}+(g \circ \nabla u)(t)\right\}+\frac{1}{p} I(u(t))+\frac{1}{2}\left\|u_{t}(t)\right\|_{2}^{2} \\
& \geq \frac{p-2}{2 p}\left\{\left(1-\int_{0}^{t} g(s) d s\right)\|\nabla u(t)\|_{2}^{2}+(g \circ \nabla u)(t)\right\}+\frac{1}{2}\left\|u_{t}(t)\right\|_{2}^{2},
\end{aligned}
$$

since $I(u(t)) \geq 0$ and $(g \circ \nabla u)(t)$ are positive. Therefore

$$
\|\nabla u(t)\|_{2}^{2}+\left\|u_{t}(t)\right\|_{2}^{2} \leq C E(0)
$$

where $C$ is a positive constant.

For establishing the general decay rate estimate, we use the following functional:

$$
F(t):=E(t)+\epsilon_{1} \Psi(t)+\epsilon_{2} \Phi(t),
$$

where $\epsilon_{1}$ and $\epsilon_{2}$ are positive constants and

$$
\begin{aligned}
& \Psi(t):=\xi(t) \int_{\Omega} u u_{t} d x, \\
& \Phi(t):=\xi(t) \int_{\Omega}\left(\Delta u-u_{t}\right) \int_{0}^{t} g(t-\tau)(u(t)-u(\tau)) d \tau d x .
\end{aligned}
$$

Lemma 4.3 For $\epsilon_{1}$ and $\epsilon_{2}$ small enough, we have

$$
\alpha_{1} F(t) \leq E(t) \leq \alpha_{2} F(t)
$$

holds, where $\alpha_{1}$ and $\alpha_{2}$ are positive constants.

Proof By straightforward computations, we obtain

$$
\begin{aligned}
F(t) \leq & E(t)+\frac{\epsilon_{1}}{2} \xi(t) \int_{\Omega}|u|^{2} d x+\frac{\epsilon_{1}}{2} \xi(t) \int_{\Omega}\left|u_{t}\right|^{2} d x+\frac{\epsilon_{2}}{2} \xi(t) \int_{\Omega}\left|u_{t}\right|^{2} d x \\
& +\frac{\epsilon_{2}}{2} C_{*}^{2}(1-l) \xi(t)(g \circ \nabla u)+\frac{\epsilon_{2}}{2} \xi(t) \int_{\Omega}|\nabla u|^{2} d x+\frac{\epsilon_{2}}{2}(1-l) \xi(t)(g \circ \nabla u) \\
\leq & E(t)+\frac{1}{2}\left(\epsilon_{1}+\epsilon_{2}\right) \xi(t) \int_{\Omega}\left|u_{t}\right|^{2} d x+\frac{1}{2}\left(\epsilon_{1} C_{*}^{2}+\epsilon_{2}\right) \xi(t) \int_{\Omega}|\nabla u|^{2} d x
\end{aligned}
$$




$$
\begin{aligned}
& +\frac{\epsilon_{2}}{2}\left(C_{*}^{2}+1\right)(1-l) \xi(t)(g \circ \nabla u)(t) \\
\leq & \frac{1}{2}\left[1+\left(\epsilon_{1}+\epsilon_{2}\right) M\right]\left\|u_{t}\right\|_{2}^{2}+\frac{1}{2}\left[l+\left(\epsilon_{1} C_{*}^{2}+\epsilon_{2}\right) M\right]\|\nabla u\|_{2}^{2} \\
& +\left[\frac{1}{2}+\frac{\epsilon_{2}}{2}\left(C_{*}^{2}+1\right) M(1-l)\right](g \circ \nabla u)(t)-\frac{1}{p}\|u(t)\|_{p}^{p} \\
\leq & \frac{1}{\alpha_{1}} E(t) .
\end{aligned}
$$

Similarly, we have

$$
\begin{aligned}
F(t) \geq & E(t)-\frac{1}{2}\left(\epsilon_{1}+\epsilon_{2}\right) \xi(t) \int_{\Omega}\left|u_{t}\right|^{2} d x-\frac{1}{2}\left(\epsilon_{1} C_{*}^{2}+\epsilon_{2}\right) \xi(t) \int_{\Omega}|\nabla u|^{2} d x \\
& -\frac{\epsilon_{2}}{2}\left(C_{*}^{2}+1\right)(1-l) \xi(t)(g \circ \nabla u)(t) \\
\geq & {\left[\frac{l}{2}-\frac{1}{2} M\left(\epsilon_{1} C_{*}^{2}+\epsilon_{2}\right)\right]\|\nabla u\|_{2}^{2}+\left(\frac{1}{2}-\frac{\epsilon_{1}+\epsilon_{2}}{2} M\right)\left\|u_{t}\right\|_{2}^{2} } \\
& +\left[\frac{1}{2}-\frac{\epsilon_{2}}{2} M\left(C_{*}^{2}+1\right)(1-l)\right](g \circ \nabla u)(t)-\frac{1}{p}\|u(t)\|_{p}^{p} \\
\geq & \frac{1}{\alpha_{2}} E(t)
\end{aligned}
$$

for $\epsilon_{1}$ and $\epsilon_{2}$ small enough.

Lemma 4.4 If (H1), (H2) hold and $u$ is the solution of $(1.1)$, let $\left(u_{0}, u_{1}\right) \in H_{0}^{1}(\Omega) \times L^{2}(\Omega)$ be given, then the functional

$$
\Psi(t):=\xi(t) \int_{\Omega} u u_{t} d x
$$

satisfies

$$
\begin{aligned}
\Psi^{\prime}(t) \leq & \left(1+\frac{1+k}{4 \alpha}\right) \xi(t)\left\|u_{t}\right\|_{2}^{2}+\left\{-\frac{l}{2}+\frac{1}{4 \alpha}+(1+k) \alpha C_{*}^{2}\right\} \xi(t)\|\nabla u\|_{2}^{2} \\
& +\frac{1-l}{2 l} \xi(t)(g \circ \nabla u)(t)+\alpha \xi(t)\left\|\nabla u_{t}\right\|_{2}^{2}+\xi(t)\|u\|_{p}^{p} .
\end{aligned}
$$

Proof Taking a time derivative of (4.8) and using equation (1.1), we have

$$
\begin{aligned}
\Psi^{\prime}(t)= & \xi(t) \int_{\Omega}\left|u_{t}\right|^{2} d x+\xi(t) \int_{\Omega} u u_{t t} d x+\xi^{\prime}(t) \int_{\Omega} u u_{t} d x \\
= & \xi(t)\left(\left\|u_{t}\right\|_{2}^{2}-\|\nabla u\|_{2}^{2}+\int_{\Omega} \nabla u(t) \int_{0}^{t} g(t-\tau) \nabla u(\tau) d \tau d x\right. \\
& \left.-\int_{\Omega} \nabla u_{t} \nabla u d x-\int_{\Omega} u_{t} u d x+\|u\|_{p}^{p}\right)+\xi^{\prime}(t) \int_{\Omega} u u_{t} d x .
\end{aligned}
$$


Now, we estimate the third term on the right-hand side of (4.10) as follows:

$$
\begin{aligned}
& \int_{\Omega} \nabla u(t) \int_{0}^{t} g(t-\tau) \nabla u(\tau) d \tau d x \\
& \quad \leq \frac{1}{2} \int_{\Omega}|\nabla u|^{2} d x+\frac{1}{2} \int_{\Omega}\left(\int_{0}^{t} g(t-\tau)|\nabla u(\tau)| d \tau\right)^{2} d x \\
& \quad \leq \frac{1}{2} \int_{\Omega}|\nabla u|^{2} d x+\frac{1}{2} \int_{\Omega}\left(\int_{0}^{t} g(t-\tau)(|\nabla u(\tau)-\nabla u(t)|+|\nabla u(t)|) d \tau\right)^{2} d x .
\end{aligned}
$$

We then use Young's inequality and (H1) to obtain, for $\forall \eta>0$,

$$
\begin{aligned}
\int_{\Omega} & \left(\int_{0}^{t} g(t-\tau)(|\nabla u(\tau)-\nabla u(t)|+|\nabla u(t)|) d \tau\right)^{2} d x \\
\leq & \int_{\Omega}\left(\int_{0}^{t} g(t-\tau)|\nabla u(\tau)-\nabla u(t)| d \tau\right)^{2} d x+\int_{\Omega}\left(\int_{0}^{t} g(t-\tau)|\nabla u(t)| d \tau\right)^{2} d x \\
& +2 \int_{\Omega}\left(\int_{0}^{t} g(t-\tau)|\nabla u(\tau)-\nabla u(t)| d \tau\right)\left(\int_{0}^{t} g(t-\tau)|\nabla u(t)| d \tau\right) d x \\
\leq & (1+\eta) \int_{\Omega}\left(\int_{0}^{t} g(t-\tau)|\nabla u(t)| d \tau\right)^{2} d x \\
& +\left(1+\frac{1}{\eta}\right) \int_{\Omega}\left(\int_{0}^{t} g(t-\tau)|\nabla u(\tau)-\nabla u(t)| d \tau\right)^{2} d x \\
\leq & \left(1+\frac{1}{\eta}\right)(1-l)(g \circ \nabla u)(t)+(1+\eta)(1-l)^{2} \int_{\Omega}|\nabla u(t)|^{2} d x
\end{aligned}
$$

By using Young's and Poincaré's inequalities and for $\forall \alpha>0$, we have

$$
\begin{aligned}
& \int_{\Omega} \nabla u_{t} \nabla u d x \leq \alpha\left\|\nabla u_{t}\right\|_{2}^{2}+\frac{1}{4 \alpha}\|\nabla u\|_{2}^{2}, \\
& \int_{\Omega} u_{t} u d x \leq \alpha C_{*}^{2}\|\nabla u\|_{2}^{2}+\frac{1}{4 \alpha}\left\|u_{t}\right\|_{2}^{2} .
\end{aligned}
$$

Combining (4.10)-(4.14) yields

$$
\begin{aligned}
\Psi^{\prime}(t) \leq & \left(1+\frac{1}{4 \alpha}+\left|\frac{\xi^{\prime}(t)}{\xi(t)}\right| \frac{1}{4 \alpha}\right) \xi(t)\left\|u_{t}\right\|_{2}^{2} \\
& +\left\{-\frac{1}{2}+\frac{1}{2}(1+\eta)(1-l)^{2}+\frac{1}{4 \alpha}+\left(1+\left|\frac{\xi^{\prime}(t)}{\xi(t)}\right|\right) \alpha C_{*}^{2}\right\} \xi(t)\|\nabla u\|_{2}^{2} \\
& +\frac{1}{2}\left(1+\frac{1}{\eta}\right)(1-l) \xi(t)(g \circ \nabla u)(t)+\alpha \xi(t)\left\|\nabla u_{t}\right\|_{2}^{2}+\xi(t)\|u\|_{p}^{p} .
\end{aligned}
$$

By choosing $\eta=l /(1-l)$ and using (H2), we can get (4.9).

Lemma 4.5 Assume that (H1), $(\mathrm{H} 2)$ hold and $\left(u_{0}, u_{1}\right) \in H_{0}^{1}(\Omega) \times L^{2}(\Omega)$ is given. If $u$ is the solution of (1.1), then the functional

$$
\Phi(t):=\xi(t) \int_{\Omega}\left(\Delta u-u_{t}\right) \int_{0}^{t} g(t-\tau)(u(t)-u(\tau)) d \tau d x
$$


satisfies

$$
\begin{aligned}
\Phi^{\prime}(t) \leq & \delta\left\{k+2(1-l)^{2}+3+C_{*}^{2(p-1)}\left(\frac{2 p}{l(p-2)} E(0)\right)^{p-2}\right\} \xi(t)\|\nabla u\|_{2}^{2} \\
& +\left\{\delta(2+k)-\int_{0}^{t} g(\tau) d \tau\right\} \xi(t)\left\|u_{t}\right\|_{2}^{2}+\frac{1}{4 \delta}(1-l)^{2} \xi(t)\left\|\nabla u_{t}\right\|_{2}^{2} \\
& +(1-l)\left(\frac{k}{4 \delta}+\frac{k C_{*}^{2}}{4 \delta}+\frac{1}{2 \delta}+2 \delta+\frac{C_{*}^{2}}{2 \delta}\right) \xi(t)(g \circ \nabla u)(t) \\
& -\frac{g(0)}{4 \delta}\left(1+C_{*}^{2}\right) \xi(t)\left(g^{\prime} \circ \nabla u\right)(t) .
\end{aligned}
$$

Proof By taking a time derivative of (4.16) and using equation (1.1), we arrive at

$$
\begin{aligned}
\Phi^{\prime}(t)= & -\xi^{\prime}(t) \int_{\Omega} \nabla u \int_{0}^{t} g(t-\tau)(\nabla u(t)-\nabla u(\tau)) d \tau d x \\
& -\xi^{\prime}(t) \int_{\Omega} u_{t} \int_{0}^{t} g(t-\tau)(u(t)-u(\tau)) d \tau d x \\
& +\xi(t) \int_{\Omega} \nabla u \int_{0}^{t} g(t-\tau)(\nabla u(t)-\nabla u(\tau)) d \tau d x \\
& -\xi(t) \int_{\Omega} \int_{0}^{t} g(t-\tau) \nabla u(\tau) d \tau \int_{0}^{t} g(t-\tau)(\nabla u(t)-\nabla u(\tau)) d \tau d x \\
& +\xi(t) \int_{\Omega} u_{t} \int_{0}^{t} g(t-\tau)(u(t)-u(\tau)) d \tau d x \\
& -\xi(t) \int_{\Omega} u|u|^{p-2} \int_{0}^{t} g(t-\tau)(u(t)-u(\tau)) d \tau d x \\
& -\xi(t) \int_{\Omega} \nabla u \int_{0}^{t} g^{\prime}(t-\tau)(\nabla u(t)-\nabla u(\tau)) d \tau d x \\
& -\xi(t) \int_{\Omega} u_{t} \int_{0}^{t} g^{\prime}(t-\tau)(u(t)-u(\tau)) d \tau d x \\
& -\xi(t) \int_{\Omega} \nabla u \int_{0}^{t} g(t-\tau) \nabla u_{t} d \tau d x-\xi(t) \int_{0}^{t} g(\tau) d \tau\left\|u_{t}\right\|_{2}^{2} \\
:= & \xi(t)\left(\left|\frac{\xi^{\prime}(t)}{\xi(t)}\right| I_{1}+\left|\frac{\xi^{\prime}(t)}{\xi(t)}\right| I_{2}+I_{3}+I_{4}+I_{5}+I_{6}\right. \\
& \left.+I_{7}+I_{8}+I_{9}-\int_{0}^{t} g(\tau) d \tau\left\|u_{t}\right\|_{2}^{2}\right) .
\end{aligned}
$$

We will estimate $I_{j}, j=1, \ldots, 9$, on the right-hand side of (4.18). Using Young's inequality, Cauchy-Schwarz's inequality, Poincaré's inequality, (H1) and (H2), for $\forall \delta>0$, we have

$$
\begin{aligned}
I_{1} & \leq \delta \int_{\Omega}|\nabla u|^{2} d x+\frac{1}{4 \delta} \int_{\Omega}\left|\int_{0}^{t} g(t-\tau)(\nabla u(t)-\nabla u(\tau)) d \tau\right|^{2} d x \\
& \leq \delta \int_{\Omega}|\nabla u|^{2} d x+\frac{1}{4 \delta} \int_{\Omega} g(t-\tau) \int_{0}^{t} g(t-\tau)(\nabla u(t)-\nabla u(\tau))^{2} d \tau d x \\
& \leq \delta\|\nabla u\|_{2}^{2}+\frac{1-l}{4 \delta}(g \circ \nabla u)(t) .
\end{aligned}
$$




$$
\begin{aligned}
I_{2} & \leq \delta \int_{\Omega}\left|u_{t}\right|^{2} d x+\frac{1}{4 \delta} \int_{\Omega}\left|\int_{0}^{t} g(t-\tau)(u(t)-u(\tau)) d \tau\right|^{2} d x \\
& \leq \delta\left\|u_{t}\right\|_{2}^{2}+\frac{1-l}{4 \delta} C_{*}^{2}(g \circ \nabla u)(t) . \\
I_{3} & \leq \delta \int_{\Omega}|\nabla u|^{2} d x+\frac{1}{4 \delta} \int_{\Omega}\left|\int_{0}^{t} g(t-\tau)(\nabla u(t)-\nabla u(\tau)) d \tau\right|^{2} d x \\
& \leq \delta\|\nabla u\|_{2}^{2}+\frac{1-l}{4 \delta}(g \circ \nabla u)(t) .
\end{aligned}
$$

By taking $\eta=1$ in (4.12), we can get $I_{4}$ as follows:

$$
\begin{aligned}
I_{4}= & \int_{\Omega} \int_{0}^{t} g(t-\tau) \nabla u(\tau) d \tau \int_{0}^{t} g(t-\tau)(\nabla u(t)-\nabla u(\tau)) d \tau d x \\
\leq & \delta \int_{\Omega}\left|\int_{0}^{t} g(t-\tau) \nabla u(\tau) d \tau\right|^{2} d x+\frac{1}{4 \delta} \int_{\Omega}\left|\int_{0}^{t} g(t-\tau)(\nabla u(t)-\nabla u(\tau)) d \tau\right|^{2} d x \\
\leq & \frac{1}{4 \delta} \int_{\Omega}\left|\int_{0}^{t} g(t-\tau)\right| \nabla u(t)-\nabla u(\tau)|d \tau|^{2} d x+2 \delta(1-l)^{2} \int_{\Omega}|\nabla u|^{2} d x \\
& +2 \delta(1-l)(g \circ \nabla u)(t) \\
\leq & 2 \delta(1-l)^{2}\|\nabla u\|_{2}^{2}+\left(2 \delta+\frac{1}{4 \delta}\right)(1-l)(g \circ \nabla u)(t) \\
I_{5} \leq & \delta \int_{\Omega}\left|u_{t}\right|^{2} d x+\frac{1}{4 \delta} \int_{\Omega}\left|\int_{0}^{t} g(t-\tau)(u(t)-u(\tau)) d \tau\right|^{2} d x \\
\leq & \delta\left\|u_{t}\right\|_{2}^{2}+\frac{1-l}{4 \delta} C_{*}^{2}(g \circ \nabla u)(t)
\end{aligned}
$$

With the help of the inequalities mentioned above and the Sobolev embedding theorem, we infer that

$$
\begin{aligned}
I_{6} & \leq \delta \int_{\Omega}|u|^{2(p-1)} d x+\frac{1}{4 \delta} \int_{\Omega}\left|\int_{0}^{t} g(t-\tau)(u(t)-u(\tau)) d \tau\right|^{2} d x \\
& \leq \delta\|u\|_{2(p-1)}^{2(p-1)}+\frac{1-l}{4 \delta} C_{*}^{2}(g \circ \nabla u)(t) \\
& \leq \delta C_{*}^{2(p-1)}\left(\frac{2 p}{l(p-2)} E(0)\right)^{p-2}\|\nabla u\|_{2}^{2}+\frac{1-l}{4 \delta} C_{*}^{2}(g \circ \nabla u)(t) .
\end{aligned}
$$

Similarly, using $(\mathrm{H} 2)$, we obtain $I_{7}, I_{8}, I_{9}$ as follows:

$$
\begin{aligned}
I_{7} & \leq \delta \int_{\Omega}|\nabla u|^{2} d x+\frac{1}{4 \delta} \int_{\Omega}\left|\int_{0}^{t} g^{\prime}(t-\tau)(\nabla u(t)-\nabla u(\tau)) d \tau\right|^{2} d x \\
& \leq \delta \int_{\Omega}|\nabla u|^{2} d x+\frac{1}{4 \delta} \int_{\Omega} g^{\prime}(t-\tau) \int_{0}^{t} g^{\prime}(t-\tau)|\nabla u(t)-\nabla u(\tau)|^{2} d \tau d x \\
& \leq \delta\|\nabla u\|_{2}^{2}-\frac{g(0)}{4 \delta}\left(g^{\prime} \circ \nabla u\right)(t) ; \\
I_{8} & \leq \delta \int_{\Omega}\left|u_{t}\right|^{2} d x+\frac{1}{4 \delta} \int_{\Omega}\left|\int_{0}^{t} g^{\prime}(t-\tau)(u(t)-u(\tau)) d \tau\right|^{2} d x \\
& \leq \delta\left\|u_{t}\right\|_{2}^{2}-\frac{g(0)}{4 \delta} C_{*}^{2}\left(g^{\prime} \circ \nabla u\right)(t) ;
\end{aligned}
$$




$$
\begin{aligned}
I_{9} & \leq \delta \int_{\Omega}|\nabla u|^{2} d x+\frac{1}{4 \delta} \int_{\Omega}\left|\int_{0}^{t} g(t-\tau) \nabla u_{t} d \tau\right|^{2} d x \\
& \leq \delta\|\nabla u\|_{2}^{2}+\frac{1}{4 \delta}(1-l)^{2}\left\|\nabla u_{t}\right\|_{2}^{2} .
\end{aligned}
$$

Combining (4.18)-(4.27), we get estimate (4.17).

Theorem 4.6 Let $\left(u_{0}, u_{1}\right) \in H_{0}^{1}(\Omega) \times L^{2}(\Omega)$ be given, satisfying (4.1). If (H1), (H2), and (H3) hold, then, for each $t_{0}>0$, there exist strictly positive constants $K$ and $\lambda$ such that the solution of (1.1) satisfies

$$
E(t) \leq K e^{-\lambda \int_{t_{0}}^{t} \xi(t) d s}, \quad \forall t \geq t_{0} .
$$

Proof For $g$ is positive and continuous, $g(0)>0$, then for $\forall t_{0}>0$ we get

$$
\int_{0}^{t} g(\tau) d \tau \geq \int_{0}^{t_{0}} g(\tau) d \tau=g_{0}>0, \quad \forall t \geq t_{0}
$$

Taking a time derivative of (4.6), using (2.3), (4.6), (4.9), (4.17), (4.29) and Remark 2.1, we get, for $\forall t \geq t_{0}$,

$$
\begin{aligned}
F^{\prime}(t) \leq & -\left\{\epsilon_{2}\left[g_{0}-\delta(2+k)\right]-\epsilon_{1}\left(1+\frac{1+k}{4 \alpha}\right)\right\} \xi(t)\left\|u_{t}\right\|_{2}^{2} \\
& -\left\{\epsilon_{1}\left[\frac{l}{2}-\frac{1}{4 \alpha}-(1+k) \alpha C_{*}^{2}\right]-\epsilon_{2} \delta\left[k+2(1-l)^{2}+3\right.\right. \\
& \left.\left.+C_{*}^{2(p-1)}\left(\frac{2 p}{l(p-2)} E(0)\right)^{p-2}\right]\right\} \xi(t)\|\nabla u\|_{2}^{2} \\
& +\left\{\frac{\epsilon_{1}(1-l)}{2 l}+\epsilon_{2}(1-l)\left(\frac{k}{4 \delta}+\frac{k C_{*}^{2}}{4 \delta}+\frac{1}{2 \delta}+2 \delta+\frac{C_{*}^{2}}{2 \delta}\right)\right\} \xi(t)(g \circ \nabla u)(t) \\
& +\left(\frac{1}{2}-\frac{g(0)}{4 \delta}\left(1+C_{*}^{2}\right) \epsilon_{2} M\right)\left(g^{\prime} \circ \nabla u\right)(t) \\
& -\left(1-\epsilon_{1} \alpha M-\frac{\epsilon_{2}}{4 \delta}(1-l)^{2} M\right)\left\|\nabla u_{t}\right\|_{2}^{2}+\epsilon_{1} \xi(t)\|u\|_{p}^{p} .
\end{aligned}
$$

At this point we choose $\delta$ small enough such that

$$
\begin{aligned}
& \frac{g_{0}-\delta(2+k)}{1+\frac{1+k}{4 \alpha}}>\frac{1}{2} g_{0}, \\
& \frac{\delta\left[k+2(1-l)^{2}+3+C_{*}^{2(p-1)}\left(\frac{2 p}{l(p-2)} E(0)\right)^{p-2}\right]}{\frac{l}{2}-\frac{1}{4 \alpha}-(1+k) \alpha C_{*}^{2}}<\frac{1}{4} g_{0} .
\end{aligned}
$$

When $\delta$ is fixed, we choose any two positive constants $\epsilon_{1}$ and $\epsilon_{2}$ satisfying

$$
\frac{1}{4} g_{0} \epsilon_{2}<\epsilon_{1}<\frac{1}{2} g_{0} \epsilon_{2}
$$

will make

$$
\kappa_{1}:=\epsilon_{2}\left[g_{0}-\delta(2+k)\right]-\epsilon_{1}\left(1+\frac{1+k}{4 \alpha}\right)>0
$$




$$
\begin{aligned}
\kappa_{2}:= & \epsilon_{1}\left[\frac{l}{2}-\frac{1}{4 \alpha}-(1+k) \alpha C_{*}^{2}\right] \\
& -\epsilon_{2} \delta\left[k+2(1-l)^{2}+3+C_{*}^{2(p-1)}\left(\frac{2 p}{l(p-2)} E(0)\right)^{p-2}\right]>0 .
\end{aligned}
$$

We then pick $\epsilon_{1}$ and $\epsilon_{2}$ small enough such that (4.7) and (4.31) remain valid and

$$
\begin{aligned}
\kappa_{3}:= & \left(\frac{1}{2}-\frac{g(0)}{4 \delta}\left(1+C_{*}^{2}\right) \epsilon_{2} M\right) \\
& -\left\{\frac{\epsilon_{1}(1-l)}{2 l}+\epsilon_{2}(1-l)\left(\frac{k}{4 \delta}+\frac{k C_{*}^{2}}{4 \delta}+\frac{1}{2 \delta}+2 \delta+\frac{C_{*}^{2}}{2 \delta}\right)\right\}>0, \\
\kappa_{4}:= & 1-\epsilon_{1} \alpha M-\frac{1}{4 \delta}(1-l)^{2} \epsilon_{2} M<0 .
\end{aligned}
$$

Hence

$$
\begin{aligned}
\left(\frac{1}{2}\right. & \left.-\frac{g(0)}{4 \delta}\left(1+C_{*}^{2}\right) \epsilon_{2} M\right)\left(g^{\prime} \circ \nabla u\right)(t) \\
& +\left\{\frac{\epsilon_{1}(1-l)}{2 l}+\epsilon_{2}(1-l)\left(\frac{k}{4 \delta}+\frac{k C_{*}^{2}}{4 \delta}+\frac{1}{2 \delta}+2 \delta+\frac{C_{*}^{2}}{2 \delta}\right)\right\} \xi(t)(g \circ \nabla u)(t) \\
\leq & -\kappa_{3} \xi(t)(g \circ \nabla u)(t) .
\end{aligned}
$$

For $\xi(t)$ is nonincreasing. Therefore, by using (4.7) and (4.30), we arrive at

$$
F^{\prime}(t) \leq-\gamma \xi(t) E(t) \leq-\gamma \alpha_{1} \xi(t) F(t), \quad \forall t \geq t_{0} .
$$

By integration of (4.33), we get

$$
F(t) \leq F\left(t_{0}\right) e^{-\gamma \alpha_{1} \int_{t_{0}}^{t} \xi(s) d s}, \quad \forall t \geq t_{0} .
$$

Thus (4.7) and (4.34) yield

$$
E(t) \leq \alpha_{2} F\left(t_{0}\right) e^{-\gamma \alpha_{1} \int_{t_{0}}^{t} \xi(s) d s}=K e^{-\lambda \int_{t_{0}}^{t} \xi(s) d s}, \quad \forall t \geq t_{0} .
$$

Remark 4.7 We can obtain exponential decay if $\xi(t)=a$ and polynomial decay if $\xi(t)=$ $a(1+t)^{-1}$, where $a>0$ is a constant.

Remark 4.8 For the continuity and boundedness of $E(t)$ and $\xi(t)$, estimates of (4.34) are also true for $t \in\left[0, t_{0}\right]$.

\section{Blow-up phenomenon}

In this section we state and prove the blow-up result.

Theorem 5.1 If (H1), (H2), (H3) hold, $E(0)<0$ and $\int_{0}^{\infty} g(\tau) d \tau<\frac{(p / 2)-1}{p / 2-1+(1 / 2 p)}$, then the solution of (1.1) blows up in finite time. 
Proof For the definition of $H(t)$, we have

$$
H^{\prime}(t)=-\frac{1}{2}\left(g^{\prime} \circ \nabla u\right)(t)+\frac{1}{2} g(t)\|\nabla u(t)\|_{2}^{2}+\left\|\nabla u_{t}\right\|_{2}^{2}+\left\|u_{t}\right\|_{2}^{2} \geq 0
$$

and

$$
0<H(0) \leq H(t) \leq \frac{1}{p}\|u\|_{p}^{p} .
$$

Furthermore, we define

$$
L(t)=H^{1-\alpha}(t)+\epsilon \int_{\Omega} u u_{t} d x,
$$

where $\epsilon$ is a small constant and will be chosen later, $0<\alpha<\frac{p-2}{2 p}$.

By taking a time derivative of (5.2), we get

$$
\begin{aligned}
L^{\prime}(t)= & (1-\alpha) H^{-\alpha}(t) H^{\prime}(t)+\epsilon \int_{\Omega}\left|u_{t}\right|^{2} d x+\epsilon \int_{\Omega} u u_{t t} d x \\
= & (1-\alpha) H^{-\alpha}(t) H^{\prime}(t)+\epsilon \int_{\Omega}\left|u_{t}\right|^{2} d x-\epsilon \int_{\Omega}|\nabla u|^{2} d x-\epsilon \int_{\Omega} u_{t} u d x \\
& +\epsilon \int_{\Omega} \nabla u \int_{0}^{t} g(t-\tau) \nabla u(\tau) d \tau d x-\epsilon \int_{\Omega} \nabla u \cdot \nabla u_{t} d x+\epsilon \int_{\Omega}|u|^{p} d x .
\end{aligned}
$$

Using Young's and Schwarz's inequalities, we obtain

$$
\begin{aligned}
& \int_{\Omega} \nabla u \int_{0}^{t} g(t-\tau) \nabla u(\tau) d \tau d x \\
& \quad=\int_{\Omega} \nabla u \int_{0}^{t} g(t-\tau)(\nabla u(\tau)-\nabla u(t)) d \tau d x+\int_{\Omega} \nabla u \int_{0}^{t} g(t-\tau) \nabla u(t) d \tau d x \\
& \quad \geq-\delta\|\nabla u\|_{2}^{2}-\frac{1}{4 \delta}\left(\int_{0}^{t} g(\tau) d \tau\right)(g \circ \nabla u)(t)+\left(\int_{0}^{t} g(\tau) d \tau\right)\|\nabla u\|_{2}^{2}, \\
& \int_{\Omega} \nabla u \cdot \nabla u_{t} d x \geq-\gamma\left\|\nabla u_{t}\right\|_{2}^{2}-\frac{1}{4 \gamma}\|\nabla u\|_{2}^{2}, \\
& \int_{\Omega} u_{t} u d x \geq-\frac{\delta^{2}}{2}\|u\|_{2}^{2}-\frac{\delta^{-2}}{2}\left\|u_{t}\right\|_{2}^{2},
\end{aligned}
$$

where $\delta$ and $\gamma$ are positive constants.

Inserting (5.4), (5.5), and (5.6) into (5.3), we deduce

$$
\begin{aligned}
L^{\prime}(t) \geq & (1-\alpha) H^{-\alpha}(t) H^{\prime}(t)+\epsilon\left(1-\frac{\delta^{-2}}{2}\right)\left\|u_{t}\right\|_{2}^{2}-\gamma \epsilon\left\|\nabla u_{t}\right\|_{2}^{2} \\
& +\epsilon\left(-1-\delta+\int_{0}^{t} g(\tau) d \tau-\frac{1}{4 \gamma}\right)\|\nabla u\|_{2}^{2}-\frac{\epsilon}{4 \delta} \int_{0}^{t} g(\tau) d \tau(g \circ \nabla u)(t) \\
& -\frac{\delta^{2}}{2} \epsilon\|u\|_{2}^{2}+\epsilon\|u\|_{p}^{p} .
\end{aligned}
$$

From (2.3), we know that

$$
-\left\|\nabla u_{t}\right\|_{2}^{2} \geq E^{\prime}(t)=-H^{\prime}(t) .
$$


If we set $\delta^{2}=k H^{\alpha}(t), \delta^{-2}=k^{-1} H^{-\alpha}(t)$, where $k>0$, then we have

$$
-\frac{\delta^{2}}{2} \epsilon\|u\|_{2}^{2}=-\frac{\epsilon}{2} k H^{\alpha}(t)\|u\|_{2}^{2}
$$

Using (2.1) and (5.1), we obtain

$$
H^{\alpha}(t)\|u\|_{2}^{2} \leq C\left(\frac{1}{p}\right)^{\alpha}\|u\|_{p}^{2+\alpha p},
$$

where we let $2 \leq 2+\alpha p \leq p$, then $0<\alpha \leq \frac{p-2}{p}$.

Using Corollary 2.4 and (2.2), inserting (5.10) and (5.8) into (5.7), we obtain

$$
\begin{aligned}
L^{\prime}(t) \geq[ & \left.(1-\alpha) H^{-\alpha}(t)-\gamma \epsilon\right] H^{\prime}(t)+\epsilon\left(p-\frac{k C}{2}\left(\frac{1}{p}\right)^{\alpha}\right) H(t) \\
& +\epsilon\left\{\frac{p-2}{2}\left(1-\int_{0}^{t} g(\tau) d \tau\right)-\delta-\frac{1}{4 \gamma}-\frac{k C}{2}\left(\frac{1}{p}\right)^{\alpha}\right\}\|\nabla u\|_{2}^{2} \\
& +\epsilon\left\{\frac{p}{2}-\frac{1}{4 \delta} \int_{0}^{t} g(\tau) d \tau-\frac{k C}{2}\left(\frac{1}{p}\right)^{\alpha}\right\}(g \circ \nabla u)(t) \\
+ & \epsilon\left\{\frac{p+2}{2}-\frac{1}{2 k} H^{-\alpha}(t)-\frac{k C}{2}\left(\frac{1}{p}\right)^{\alpha}\right\}\left\|u_{t}\right\|_{2}^{2} .
\end{aligned}
$$

By using the hypothesis in Theorem 5.1 and taking $k, \gamma$ and $\delta$ suitable such that

$$
\begin{aligned}
& \frac{p-2}{2}\left(1-\int_{0}^{t} g(\tau) d \tau\right)-\delta-\frac{1}{4 \gamma}-\frac{k C}{2}\left(\frac{1}{p}\right)^{\alpha}>0, \\
& \frac{p}{2}-\frac{1}{4 \delta} \int_{0}^{t} g(\tau) d \tau-\frac{k C}{2}\left(\frac{1}{p}\right)^{\alpha}>0, \\
& \frac{p+2}{2}-\frac{1}{2 k} H^{-\alpha}(t)-\frac{k C}{2}\left(\frac{1}{p}\right)^{\alpha}>0 .
\end{aligned}
$$

When $k, \gamma$ is fixed, we choose $\epsilon$ small enough such that

$$
(1-\alpha) H^{-\alpha}(t)-\gamma \epsilon>0, \quad L(0)=H^{1-\alpha}(0)+\epsilon \int_{\Omega} u_{0} u_{1} d x>0
$$

Then we can deduce that

$$
L^{\prime}(t) \geq C\left[H(t)+\left\|u_{t}\right\|_{2}^{2}+\|\nabla u\|_{2}^{2}+(g \circ \nabla u)(t)\right] .
$$

By using Hölder's and Young's inequalities, we get

$$
\begin{aligned}
\left|\int_{\Omega} u u_{t} d x\right|^{1 /(1-\alpha)} & \leq\|u\|_{2}^{1 /(1-\alpha)}\left\|u_{t}\right\|_{2}^{1 /(1-\alpha)} \\
& \leq C\|u\|_{p}^{1 /(1-\alpha)}\left\|u_{t}\right\|_{2}^{1 /(1-\alpha)} \\
& \leq C\left(\|u\|_{p}^{s}+\left\|u_{t}\right\|_{2}^{2}\right) \\
& \leq C\left(H(t)+\left\|u_{t}\right\|_{2}^{2}+(g \circ \nabla u)(t)+\|\nabla u\|_{2}^{2}\right)
\end{aligned}
$$


where $2 \leq s:=\frac{2}{1-2 \alpha} \leq p$, then $0<\alpha<\frac{p-2}{2 p}$. Hence

$$
\begin{aligned}
L^{1 /(1-\alpha)}(t) & =\left(H^{1-\alpha}(t)+\epsilon \int_{\Omega} u u_{t} d x\right)^{1 /(1-\alpha)} \\
& \leq 2^{1 /(1-\alpha)}\left(H(t)+\left|\int_{\Omega} u u_{t} d x\right|^{1 /(1-\alpha)}\right) \\
& \leq C\left(H(t)+\left\|u_{t}\right\|_{2}^{2}+\|\nabla u\|_{2}^{2}+(g \circ \nabla u)(t)\right) .
\end{aligned}
$$

Then we can get

$$
L^{\prime}(t) \geq \lambda L^{\frac{1}{1-\alpha}}(t), \quad t>0
$$

Therefore

$$
L(t) \geq\left(L^{\frac{-\alpha}{1-\alpha}}(0)+\frac{-\alpha}{1-\alpha} \lambda t\right)^{-\frac{1-\alpha}{\alpha}} .
$$

So $L(t)$ tends to infinity when $t$ tends to $(1-\alpha) /\left(\alpha \lambda L^{\frac{\alpha}{1-\alpha}}(0)\right)$.

To get another blow-up result, we first give the following lemma.

Lemma 5.2 If (H1), (H2) hold, assume further that

$$
\left\|u_{0}\right\|_{p}>\lambda_{0} \equiv B_{0}^{\frac{-2}{p-2}}, \quad E(0)<E_{0}=\left(\frac{1}{2}-\frac{1}{p}\right) B_{0}^{\frac{-2 p}{p-2}}
$$

Then

$$
\|u\|_{p}>\lambda_{0}, \quad\|\nabla u\|_{2}>B_{0}^{\frac{-p}{p-2}}, \quad \forall t \geq 0
$$

where $B_{0}=\frac{B}{l^{1 / 2}}$ for $\|u\|_{p} \leq B\|\nabla u\|_{2}$.

Proof By using (2.2) and the hypothesis, we obtain

$$
\begin{aligned}
E(t) & =\frac{1}{2}\left\|u_{t}(t)\right\|_{2}^{2}+\frac{1}{2}\left(1-\int_{0}^{t} g(\tau) d \tau\right)\|\nabla u(t)\|_{2}^{2}+\frac{1}{2}(g \circ \nabla u)(t)-\frac{1}{p}\|u(t)\|_{p}^{p} \\
& \geq \frac{1}{2}\left(1-\int_{0}^{t} g(\tau) d \tau\right)\|\nabla u(t)\|_{2}^{2}-\frac{1}{p}\|u(t)\|_{p}^{p} \\
& \geq \frac{l}{2}\|\nabla u(t)\|_{2}^{2}-\frac{1}{p}\|u(t)\|_{p}^{p} \\
& \geq \frac{1}{2 B_{0}^{2}}\|u(t)\|_{p}^{2}-\frac{1}{p}\|u(t)\|_{p}^{p}
\end{aligned}
$$

We set $h(\xi)=\frac{1}{2 B_{0}^{2}} \xi^{2}-\frac{1}{p} \xi^{p}, \xi>0$. Then $h(\xi)$ satisfies

- $h(\xi)$ is strictly increasing on $\left[0, \lambda_{0}\right)$;

- $h(\xi)$ takes its maximum value $\left(\frac{1}{2}-\frac{1}{p}\right) B_{0}^{\frac{-2 p}{p-2}}$ at $\lambda_{0}$;

- $h(\xi)$ is strictly decreasing on $\left(\lambda_{0}, \infty\right)$. 
Since $E_{0}>E(0) \geq E(t) \geq h\left(\|u\|_{p}\right)$ for $\forall t \geq 0$, there is no time $t$ such that $\|u\|_{p}=\lambda_{0}$. By the continuity, we obtain

$$
\|u(\cdot, t)\|_{p}>\lambda_{0}=B_{0}^{\frac{-2}{p-2}}, \quad \forall t \geq 0 .
$$

Then

$$
\|\nabla u(\cdot, t)\|_{2} \geq \frac{1}{l^{1 / 2} B_{0}}\|u(\cdot, t)\|_{p}>\frac{1}{l^{1 / 2}} B_{0}^{\frac{-p}{p-2}}>B_{0}^{\frac{-p}{p-2}}
$$

This completes the proof.

Theorem 5.3 If that (H1), (H2), and (H3) hold, suppose further that

$$
\int_{0}^{\infty} g(\tau) d \tau<\frac{(p / 2)-1}{p / 2-1+(1 / 2 p)}
$$

$\left\|u_{0}\right\|_{p}>\lambda_{0}$ and $E(0) \leq E_{0}$. Then the solution of (1.1) blows up in finite time.

Proof Set $G(t)=E_{0}+H(t)$, then

$$
G^{\prime}(t)=-\frac{1}{2}\left(g^{\prime} \circ \nabla u\right)(t)+\frac{1}{2} g(t)\|\nabla u(t)\|_{2}^{2}+\left\|\nabla u_{t}\right\|_{2}^{2}+\left\|u_{t}\right\|_{2}^{2} \geq 0
$$

from which we obtain

$$
\begin{aligned}
0<G(t) & =E_{0}+H(t) \\
& =\left(\frac{1}{2}-\frac{1}{p}\right) B_{0}^{\frac{-2 p}{p-2}}+H(t) \\
& \leq\left(\frac{1}{2}-\frac{1}{p}\right)\|\nabla u\|_{2}^{2}+H(t) \\
& \leq C\left(\|\nabla u\|_{2}^{2}+H(t)\right) .
\end{aligned}
$$

By using Lemma 5.2, we have

$$
\begin{aligned}
0< & G(t) \\
= & E_{0}-\frac{1}{2}\left\|u_{t}\right\|_{2}^{2}-\frac{1}{2}\left(1-\int_{0}^{t} g(\tau) d \tau\right)\|\nabla u(t)\|_{2}^{2} \\
& -\frac{1}{2}(g \circ \nabla u)(t)+\frac{1}{p}\|u(t)\|_{p}^{p} \\
\leq & E_{0}-\frac{1}{2}\left(1-\int_{0}^{t} g(\tau) d \tau\right)\|\nabla u(t)\|_{2}^{2}+\frac{1}{p}\|u(t)\|_{p}^{p} \\
\leq & \left(\frac{1}{2}-\frac{1}{p}\right) B_{0}^{\frac{-2 p}{p-2}}-\frac{l}{2}\left(\frac{1}{l^{1 / 2}}\right)^{2} B_{0}^{\frac{-2 p}{p-2}}+\frac{1}{p}\|u(t)\|_{p}^{p} \\
\leq & \frac{1}{p}\|u(t)\|_{p}^{p} .
\end{aligned}
$$


Let

$$
Q(t)=G^{1-\alpha}(t)+\epsilon \int_{\Omega} u u_{t} d x,
$$

with $\epsilon$ small to be chosen later and $0<\alpha<\frac{p-2}{2 p}$.

By the same computations as in the proof of Theorem 5.1, we can deduce that

$$
Q^{\prime}(t) \geq C\left[H(t)+\left\|u_{t}\right\|_{2}^{2}+\|\nabla u\|_{2}^{2}+(g \circ \nabla u)(t)\right] .
$$

Observing (5.13), we see that

$$
\begin{aligned}
Q^{1 /(1-\alpha)}(t) & =\left(G^{1-\alpha}(t)+\epsilon \int_{\Omega} u u_{t} d x\right)^{1 /(1-\alpha)} \\
& \leq 2^{1 /(1-\alpha)}\left(G(t)+\left|\int_{\Omega} u u_{t} d x\right|^{1 /(1-\alpha)}\right) \\
& \leq C\left(H(t)+\left\|u_{t}\right\|_{2}^{2}+\|\nabla u\|_{2}^{2}+(g \circ \nabla u)(t)\right) .
\end{aligned}
$$

Then we can obtain

$$
Q^{\prime}(t) \geq \lambda Q^{\frac{1}{1-\alpha}}(t), \quad t>0 .
$$

Therefore, we get

$$
Q(t) \geq\left(Q^{\frac{-\alpha}{1-\alpha}}(0)+\frac{-\alpha}{1-\alpha} \lambda t\right)^{-\frac{1-\alpha}{\alpha}}, \quad t>0 .
$$

So $Q(t)$ tends to infinity when $t$ tends to $(1-\alpha) /\left(\alpha \lambda Q^{\frac{\alpha}{1-\alpha}}(0)\right)$.

\section{Acknowledgements}

The authors are highly thankful for the referees' valuable suggestions and for the help from Hubei Key Laboratory of Computational Science.

\section{Funding}

Not applicable.

Availability of data and materials Not applicable.

\section{Competing interests}

The authors declare that they have no competing interests.

\section{Authors' contributions}

The authors declare that the study was realized in collaboration with the same responsibility. All authors read and approved the final manuscript.

\section{Publisher's Note}

Springer Nature remains neutral with regard to jurisdictional claims in published maps and institutional affiliations. Received: 12 April 2018 Accepted: 18 September 2018 Published online: 27 September 2018 


\section{References}

1. Berrimi, S., Messaoudi, S.A.: Existence and decay of solutions of a viscoelastic equation with a nonlinear source. Nonlinear Anal. 64, 2314-2331 (2006)

2. Cavalcanti, M.M., Domingos Cavalcanti, V.N., Ferreira, J.: Existence and uniform decay for a nonlinear viscoelastic equation with strong damping. Math. Methods Appl. Sci. 24, 1043-1053 (2001)

3. Cavalcanti, M.M., Domingos Cavalcanti, V.N., Lasiecka, I.: Well-posedness and optimal decay rates for the wave equation with nonlinear boundary damping-source interaction. J. Differ. Equ. 236, 407-459 (2007)

4. Cavalcanti, M.M., Domingos Cavalcanti, V.N., Lasiecka, I., Webler, C.M.: Intrinsic decay rates for the energy of a nonlinear viscoelastic equation modeling the vibrations of thin rods with variable density. Adv. Nonlinear Anal. 6 , $121-145$ (2017)

5. Chen, H., Liu, G.W.: Global existence, uniform decay and exponential growth for a class of semilinear wave equation with strong damping. Acta Math. Sci. 35B, 41-58 (2013)

6. Gazzola, F., Squassina, M.: Global solutions and finite time blow up for damped semilinear wave equations. Ann. Inst. H. Poincaré 23, 185-207 (2006)

7. Georgiev, V., Todorova, G.: Existence of solutions of the wave equation with nonlinear damping and source terms J. Differ. Equ. 109, 295-308 (1994)

8. Guesmia, A., Messaoudi, S.A., Webler, C.M.: Well-posedness and optimal decay rates for the viscoelastic Kirchhoff equation. Bol. Soc. Parana. Mat. 35, 203-224 (2017)

9. Guo, L., Yuan, Z.Q., Liu, G.G.: Blow up and global existence for a nonlinear viscoelastic wave equation with strong damping and nonlinear damping and source terms. Appl. Math. 6, 806-816 (2015)

10. Han, X.S., Wang, M.X.: General decay of energy for a viscoelastic equation with nonlinear damping. Math. Methods Appl. Sci. 347, 806-817 (2009)

11. Ikehata, R., Suzuki, T.: Exponential decay for the solution of semilinear viscoelastic wave equations with localized damping. Electron. J. Differ. Equ. 44, 227 (2002)

12. Lions, J.L.: Quelques méthods de résolution des problémes aux limites non linéaires. Gauthier-Villars, Paris (1969)

13. Liu, W.J.: General decay of solutions to a viscoelastic wave equation with nonlinear localized damping. Ann. Acad. Sci. Fenn., Math. Diss. 34, 291-302 (2009)

14. Liu, W.J.: General decay and blow-up of solution for a quasilinear viscoelastic problem with nonlinear source. Nonlinear Anal. 73, 1890-1904 (2010)

15. Messaoudi, S.A.: Blow up and global existence in a nonlinear viscoelastic wave equation. Math. Nachr. 260, 58-66 (2003)

16. Messaoudi, S.A.: Blow-up of positive-initial-energy solutions of a nonlinear viscoelastic hyperbolic equation. J. Math Anal. Appl. 320, 902-915 (2006)

17. Messaoudi, S.A.: General decay of the solution energy in a viscoelastic equation with a nonlinear source. Nonlinear Anal. 69, 2589-2598 (2008)

18. Messaoudi, S.A.: General decay of solutions of a viscoelastic equation. J. Math. Anal. Appl. 341, 1457-1467 (2008)

19. Song, H.T.: Blow up of arbitrarily positive initial energy solutions for a viscoelastic wave equation. Nonlinear Anal., Real World Appl. 26, 306-314 (2015)

20. Song, H.T., Zhong, C.K.: Blow-up of solutions of a nonlinear viscoelastic wave equation. Nonlinear Anal., Real World Appl. 26, 3877-3883 (2010)

21. Vitillaro, E.: Global existence for the wave equation with nonlinear boundary damping and source terms. J. Differ. Equ $186,259-298(2002)$

\section{Submit your manuscript to a SpringerOpen ${ }^{\circ}$ journal and benefit from:}

- Convenient online submission

- Rigorous peer review

- Open access: articles freely available online

- High visibility within the field

- Retaining the copyright to your article

Submit your next manuscript at $>$ springeropen.com 